Ann. Génét. Sél. anim., I974, 6 (I), I59-I68.

\title{
LES ANNALES DE GÉNÉTIQUE ET DE SÉLECTION ANIMALE : UN BILAN DES GINQ PREMIÈRES ANNÉES
}

\author{
J.-J. LAUVERGNE et H. K. GERINGER*
}

Laboratoire de Génétique factorielle,

Centre national de Recherches zootechniques, I. N. R. A., 78350 Jouy en Josas

* Académie agronomique, 7, rue Kozuchowska, Wroclaw (Pologne)

\section{RÉSUMÉ}

Fondées en 1969, les Annales de Génétique et de Sélection animale (A. G. S. A.) font paraître un volume annuel en 4 livraisons. Dans chaque volume on dénombre en moyenne 40 articles scientifiques d'une longueur de II pages. Ce sont surtout des mémoires originaux (82 p. Ioo).

Avec 27 p. Ioo des articles qui lui sont consacrés, la Poule est l'espèce la mieux étudiée, viennent ensuite le Bauf (24 p. Ioo des articles), le Porc (ro p. roo), la Chèvre (9 p. roo), le Mouton ( $6 \mathrm{p}$. Ioo). On dénombre également $17 \mathrm{p}$. Ioo d'articles consacrés à la méthodologie génétique et seulement $7 \mathrm{p}$. Ioo d'articles s'occupant d'autres espèces animales.

La plupart des articles traitent de la sélection, utilisant soit la génétique quantitative (36 p. IOo), soit la génétique mendélienne. L'abondance des articles consacrés à cette dernière discipline (46 p. I0o) reflète l'intérêt que les sélectionneurs portent actuellement à des gènes à effets visibles chez la Poule (le gène de nanisme) et chez le Bœuf (le gène de culard). La Caryologie rassemble ro p. roo des articles et les Polymorphismes 8 p. roo. Parmi les revues actuelles, les A. G. S. A. sont celles qui publient annuellement le plus grand nombre d'articles consacrés à l'amélioration génétique des espèces domestiques.

Les A. G. S. A. sont éditées par l'Institut national de la Recherche agronomique (I. N. R. A.) et leur Comité de rédaction est recruté parmi les chercheurs du Département de Génétique animale qui a fourni également 21 p. Ioo des I 52 auteurs déjà recensés et environ 60 p. roo des articles. On compte au total 52 p. roo d'auteurs français. 82 p. Ioo des articles sont écrits en français, I6 p. Ioo en anglais et 2 p. Ioo en allemand.

Les abonnés payant se recrutent surtout en France (3o p. Ioo) et dans la C. E. E. (24 p. 10o). Le monde anglophone, l'Asie et l'Afrique sont encore mal couverts.

\section{PRÉSENTATION DE LA REVUE}

Les premiers jalons en vue de créer les Annales de Génétique et de Sélection animale (A. G. S. A.) ont été posés auprès de la Direction de l'Institut national de la Recherche agronomique (I. N. R. A.), au début de 1967. L'autorisation a été obtenue en octobre 1968. La première livraison est parue en septembre 1969 . 
Les A. G. S. A. sont la troisième des revues de l'I. N. R. A., consacrées à l'amélioration de la productivité des animaux. Elles sont venues après les Annales de Zootechnie (A. Z.), fondées en $195 \mathrm{I}$ et les Annales de Biologie animale, Biochimie, Biophysiques (A. B. A. B. B.) fondées en 196r. Depuis, une nouvelle revue consacrée, elle aussi, aux animaux a vu le jour, en I970: les Annales de Recherches vétérinaires.

La première des revues évoquées ici (A. Z.) a suffi pendant une dizaine d'années à recueillir les articles de toutes les disciplines animales de l'I. N. R. A. Les A. B. A. B. B., lors de leur fondation, se proposaient d'accueillir les travaux plus fondamentaux. Cette revue avait atteint son but après quelques années de fonctionnement pour les disciplines de Nutrition et de Physiologie animales au moment même où les jeunes chercheurs du Département de Génétique animale de l'I. N. R. A., commençaient à assurer une production scientifique assez régulière et abondante pour remplir les colonnes d'une revue de taille moyenne. Plutôt que d'augmenter le volume des revues déjà existantes en diminuant leur spécialisation, la Direction de l'I. N. R. A. a préféré créer une revue nouvelle, ce qui correspondait au vœu des chercheurs du Département de Génétique animale.

A l'instar des douze autres séries d'Annales éditées par l'I. N. R. A., les A. G. S. A., paraissent quatre fois l'an, par fascicule de Ioo à 150 pages imprimées (de justification $12,5 \mathrm{~cm} \times 19 \mathrm{~cm}$ ) qui sont regroupés en un volume annuel. Les A. G. S. A. figurent dans Current Contents et sont également indexées dans Chemical Abstracts et dans Biological Abstracts.

L'actuel Comité de Rédaction est constitué par les responsables des divers Laboratoires et Stations du Département de Génétique animale de l'I. N. R. A., sous la présidence de B. Vissac, chef du Département, assisté de P. MÉrat, Directeur du Laboratoire de Génétique factorielle. Le secrétariat de rédaction est assuré par J.-J. Lauvergne. La Direction du Comité de rédaction, de même que la Rédaction ont leur siège au Centre national de Recherches zootechniques (C. N. R. Z.) à Jouy en Josas près de Paris.

L'impression et la diffusion des A. G. S. A. sont assurées, comme celles des autres séries d'Annales de l'I. N. R. A. par le Service des Publications de l'I. N. R. A. installé à Versailles, dans le périmètre du Centre national de Recherches agronomiques.

\section{BILAN DES ARTICLES PUBLIÉS SELON LEUR CATÉGORIE}

Dans toutes les séries d'Annales de l'I. N. R. A., on tient compte de la recommandation de l'UNESCO relative à la classification des articles scientifiques. A côté des articles de fond que sont les mémoires originaux place est faite pour les deux rubriques " Notes " et " Revues bibliographiques $\gg$.

En ce qui concerne les " Publications provisoires ou courtes notes " les A. G. S. A. distinguent en fait deux catégories : les notes qui sont référencées dans chaque fascicule et celles qui sont regroupées dans les comptes rendus de colloques ou de séminaires, généralement plus courtes et non référencées. Elles correspondent à ce que les anglo-saxons nomment les "abstracts ". A l'heure où les systèmes de documentations se contentent souvent de diffuser les tables des matières, cela constitue un handicap certain pour les auteurs de ces abstracts.

Par ailleurs, les A. G. S. A. ont publié non seulement des abstracts de communications à des colloques ou séminaires, mais également des rapports généraux présentés à des colloques et séminaires. Or, parfois, ces rapports invités, conçus pour faire le point sur une question à l'ordre du jour, ne rentrent pas exactement dans la catégorie " Exposés de mise au point " du guide de l'UNESCO dont les exigences sont plus strictes.

Avec ces deux réserves (omission des abstracts ( ${ }^{1}$ ) et assimilation abusive de certains textes, à la vérité peu nombreux) nous présentons dans le tableau I une classification des articles scien-

( $\left.{ }^{1}\right)$ C'est ainsi que, dans la suite de l'article on emploiera le terme " référencé " pour qualifier les articles et auteurs figurant dans la table des matières de la revue. 


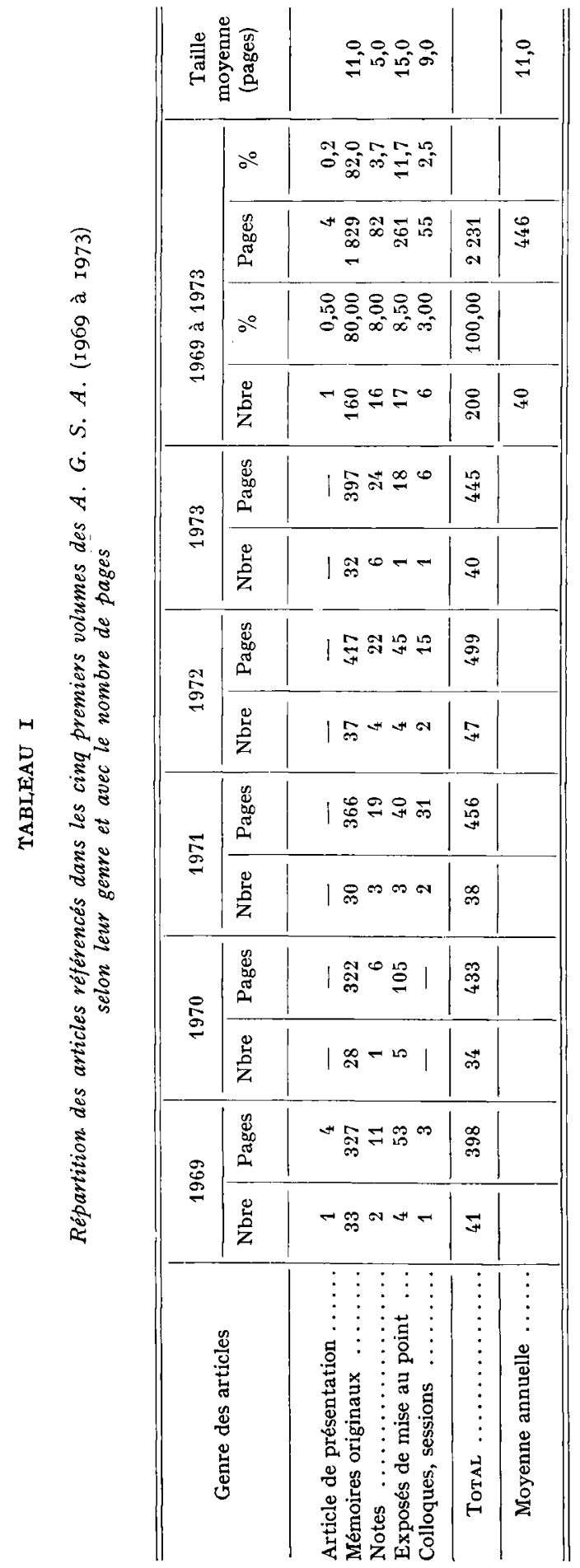


tifiques selon les catégories prescrites par l'UNESCO, en ajoutant une rubrique pour les articles qui réunissent les abstracts d'une manifestation scientifique et une rubrique pour les articles de présentation.

\section{BILAN PAR ESPÈCE E'T PAR DISCIPLINE}

Dans le tableau 2 on a ventilé les articles scientifiques référencés et signés selon l'espèce à laquelle ils se rapportent, les articles de méthodologie étant mis à part.

\section{TABLEAU 2}

Ventilation des articles scientifiques référencés dans A. G. S. A. (vol. I à 5) selon les sujets (Quelques articles (3) traitant de plusieurs espèces ont été comptés plusieurs fois)

\begin{tabular}{|c|c|c|c|c|c|c|c|}
\hline & \multirow{2}{*}{1969} & \multirow{2}{*}{1970} & \multirow{2}{*}{1971} & \multirow{2}{*}{1972} & \multirow{2}{*}{1973} & \multicolumn{2}{|c|}{ Total } \\
\hline & & & & & & Nombre & $\%$ \\
\hline Méthodologie génétique $\ldots \ldots \ldots$ & 5 & 6 & 10 & 5 & 8 & 34 & 17,3 \\
\hline Bœuf $\ldots \ldots \ldots \ldots \ldots \ldots$ & 7 & 6 & 16 & 6 & 12 & 47 & 23,9 \\
\hline Mouton $\ldots \ldots \ldots \ldots \ldots \ldots$ & 3 & 3 & 1 & 2 & 2 & 11 & 5,6 \\
\hline Chèvre $\ldots \ldots \ldots \ldots \ldots \ldots \ldots$ & 12 & 一 & 1 & 6 & - & 19 & 9,6 \\
\hline Porc $\ldots \ldots \ldots \ldots \ldots \ldots \ldots$ & 3 & 5 & 4 & 3 & 5 & 20 & 10,2 \\
\hline Poule $\ldots \ldots \ldots \ldots \ldots \ldots \ldots$ & 8 & 9 & 1 & 24 & 10 & 52 & 26,4 \\
\hline Divers $\left.\mathbf{(}^{\mathbf{1}}\right) \ldots \ldots \ldots \ldots \ldots \ldots$ & 1 & 5 & 3 & 一 & 5 & 14 & 7,0 \\
\hline Total $\ldots \ldots \ldots \ldots$ & 39 & 34 & 36 & 46 & 42 & 197 & 100,0 \\
\hline
\end{tabular}

(1) Cheval, Chien, Chat, Chauve-Souris, Cobaye, Souris, Ver à soie, Ver de farine, Drosophile.

Pour, maintenant, classer les articles au sein de chaque espèce, on a retenu les quatre rubriques suivantes : caryologie, polymorphismes (sérologiques et biochimiques), génétique mendélienne (gènes à effets visibles), génétique quantitative. De cette manière on obtient le tableau 3 .

\section{PRINCIPAUX SUJETS SCIENTIFIQUES ABORDÉS}

On aura une idée plus précise de l'orientation de la revue avec une liste des sujets abordés avec le plus d'insistance au cours des cinq premières années de fonctionnement. Six semblent spécialement devoir être cités :

- Le gène de nanisme de la Poule : ses propriétés pour l'amélioration des lignées actuelles : 1: I9, I3I, I35; $2: 19,33 ; 4: 4$ I, I83, 21 7, 225, 233, 25I, 255, 27I, 28I, 297, 305, 3I I ; $5: 3$ I3, 463 ;

- Les gènes à effets visibles chez la Poule (hormis le gène de nanisme) et leurs influences sur la productivité : $1: 33,227 ; 2: 85,135,263 ; 4: 5,67.311,375,555 ; 5: 39$; 


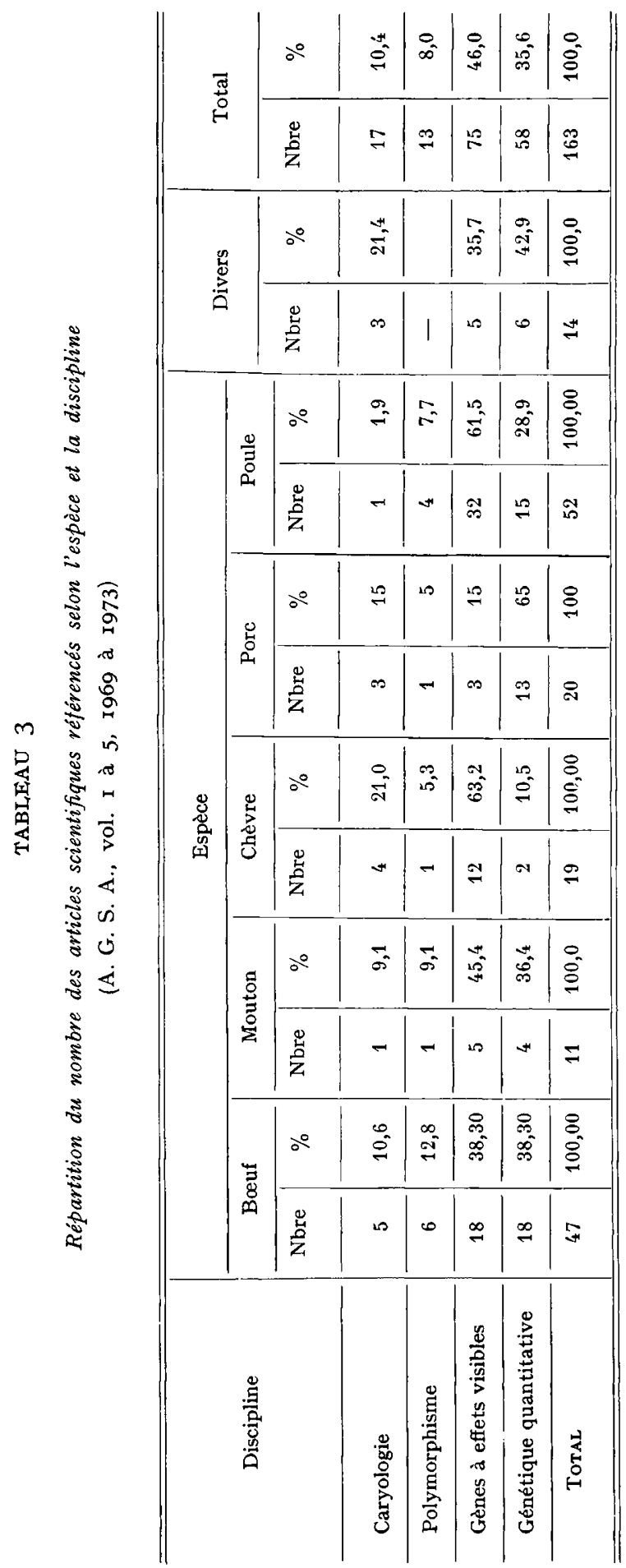


- Le gène d'absence de cornes chez la chèvre, son action sur la sexualité et la production :

$1: 34$ I, 349, 379, 383, 39I, 403, 4I3, 423, 439, 447, 465; 3:469; 4:29, 537 ;

- L'hypertrophie musculaire d'origine génétique chez les bovins : $1:$ ror ; $2: 5 ; 4: 7,88$;

$5: 23,163,441,499$;

- Optimisation des schémas d'amélioration génétique des bovins : $2: 449 ; 3: 457,509$; $5: 189,239$;

- Modèles de génétique mathématique : $1: 39,49,67,237,243 ; 2: 37,281,417,425$; $3: 255,28 I, 295,449,463,479 ; 4: 385,4$ II ; $5: 333,495$.

\section{NOMBRE E'T ORIGINE DES AUTEURS, LANGUES EMPLOYÉES}

Dans le tableau 4, figure le nombre d'auteurs différents qui ont été référencés chaque année, ainsi que le nombre d'auteurs qui font leur apparition pour la première fois au sommaire de la revue au fil des années.

\section{TABLEAU 4}

Nombre d'auteurs figurant au sommaire des cinq premiers volumes des A. G. S. A. et nombre d'auteurs nouveaux chaque année

\begin{tabular}{l|r|r|r|r|r|r}
\hline & 1969 & 1970 & 1971 & 1972 & 1973 \\
\cline { 1 - 5 } & & 37 & 33 & 47 & 52 & 54 \\
Nombre d'auteurs référencés & 37 & 21 & 31 & 34 & 29 \\
Nombre d'auteurs nouveaux & 37 & 58 & 89 & 123 & 152 \\
Nombre cumulé d'auteurs nouveaux & & & & & \\
\hline
\end{tabular}

La répartition des auteurs selon le pays où ils exercent normalement leurs activités est donnée dans le tableau 5. Pour la France on a, en outre, donné le détail des appartenances : au Département de Génétique animale de l'I. N.R. A., simplement à l'I. N. R. A. ou hors I. N. R. A.

TABLEAU 5

Origine des auteurs figurant au sommaire des cinq premiers volumes des A. G. S. A.

\begin{tabular}{|c|c|c|}
\hline & Nombre & P. 100 \\
\hline Dept. Génétique animale, I. N. R. A. . . & 36 & $23,7)$ \\
\hline I. N. R. A., hors Dept. Génét. anim. . . & 25 & $16,5\} 52,0$ \\
\hline France, hors I. N. R. A. . . . . . . . & 18 & 11,8 \\
\hline C. E. E. . . . . . . $\ldots \ldots \ldots \ldots \ldots$ & 30 & 19,7 \\
\hline Europe de l'Ouest - C. E. E. . . . . . & 9 & 5,9 \\
\hline Europe de l'Est $\ldots \ldots \ldots \ldots \ldots \ldots$ & 6 & 3,9 \\
\hline Amérique du Nord $\ldots \ldots \ldots \ldots \ldots$ & 20 & 13,2 \\
\hline Reste du monde $\ldots \ldots \ldots \ldots \ldots \ldots$ & 8 & 5,3 \\
\hline Total................... & 152 & 100,0 \\
\hline
\end{tabular}

Les statistiques du tableau 5 donnent en fait une vue biaisée de l'importance réelle des différents groupes nationaux de chercheurs qui collaborent à la revue. Un certain nombre de ces auteurs ne collaborent qu'exceptionnellement ou par le truchement de communications à des congrès. 
Pour essayer d'avoir une vision plus juste, nous avons, dans le tableau 6, choisi d'attribuer chaque article à un seul groupe d'auteurs en privilégiant hiérarchiquement dans l'ordre le Département de Génétique de l'I. N.R. A., le groupe des chercheurs de l'I. N. R. A., hors département de génétique et le groupe des chercheurs français.

TABLEAU 6

Répartition annuelle des articles scientifiques référencés selon l'origine des auteurs

\begin{tabular}{|c|c|c|c|c|c|c|c|c|c|c|c|c|}
\hline \multirow{2}{*}{$\begin{array}{l}\text { Classification } \\
\text { des articles }\end{array}$} & \multicolumn{2}{|c|}{1969} & \multicolumn{2}{|c|}{1970} & \multicolumn{2}{|c|}{1971} & \multicolumn{2}{|c|}{1972} & \multicolumn{2}{|c|}{1973} & \multicolumn{2}{|c|}{ Total } \\
\hline & Nbre & $\%$ & Nbre & $\%$ & Nbre & $\%$ & Nbre & $\%$ & Nbre & $\%$ & Nbre & $\%$ \\
\hline $\begin{array}{l}\text { Au moins un des au- } \\
\text { teurs appartient au } \\
\text { Dépt. Génét. anim. } \\
\text { I. N. R. A. }\end{array}$ & 30 & 77,0 & 20 & 60,6 & 20 & 57,1 & 22 & 48,9 & 22 & 53,7 & 114 & 59,1 \\
\hline $\begin{array}{l}\text { Au moins un des au- } \\
\text { teurs appartient à } \\
\text { l'I. N. R. A. (hors } \\
\text { Dépt. Génét. anim.) }\end{array}$ & 1 & 2,6 & 5 & 15,2 & - & & 8 & 17,8 & 5 & 12,2 & 19 & 9,8 \\
\hline $\begin{array}{l}\text { Au moins un des au- } \\
\text { teurs est français } \\
\text { hors I. N. R. A. }\end{array}$ & 2 & 5,0 & 2 & 6,0 & 3 & 8,6 & 3 & 6,7 & 1 & 2,4 & 11 & 5,7 \\
\hline $\begin{array}{l}\text { N'entrant pas dans les } \\
\text { catégories ci-dessus }\end{array}$ & 6 & 15,4 & 6 & 18,2 & 12 & 34,3 & 12 & 26,6 & 13 & 31,7 & 49 & 25,4 \\
\hline Total & 39 & 100,0 & 33 & 100,0 & 35 & 100,0 & 45 & 100,0 & 41 & 100,0 & 193 & 100,0 \\
\hline
\end{tabular}

Il est possible de publier aux A. G. S. A. dans les trois langues de la F. E. Z. : français, anglais, allemand, et le tableau 7 donne la répartition des articles selon leur idiome.

TABLEAU 7

Répartition annuelle des articles scientifiques référencés selon la langue utilisée

\begin{tabular}{|c|c|c|c|c|c|c|c|c|c|c|c|c|}
\hline \multirow{2}{*}{ Langue } & \multicolumn{2}{|c|}{1969} & \multicolumn{2}{|c|}{1970} & \multicolumn{2}{|c|}{1971} & \multicolumn{2}{|c|}{1972} & \multicolumn{2}{|c|}{1973} & \multicolumn{2}{|c|}{ Total } \\
\hline & Nbre & $\%$ & Nbre & $\%$ & Nbre & $\%$ & Nbre & $\%$ & Nbre & $\%$ & Nbre & $\%$ \\
\hline Français & 35 & 87,50 & 28 & 87,50 & 28 & 75,7 & 34 & 77,3 & 27 & 79,4 & 152 & 81,6 \\
\hline Anglais & 4 & 10,00 & 3 & 9,4 & 7 & 18,9 & 10 & 22,7 & 7 & 20,6 & 31 & 16,3 \\
\hline Allemand & 1 & 2,50 & 1 & 3,1 & 2 & 5,40 & 0 & - & 一 & - & 4 & 2,1 \\
\hline Total & 40 & 100,00 & 32 & 100,00 & 37 & 100,00 & 44 & 100,00 & 34 & 100 & 187 & 100,00 \\
\hline
\end{tabular}

Pour le détail des auteurs ayant publié dans A. G. S. A. on se reportera p. I75 de la présente livraison. 


\section{LA DIFFUSION DE LA REVUE}

Dans le tableau 8 on donne la répartition des abonnés, par nationalité.

TABLEAU 8

Répartition, par pays, des abonnements aux A. G. S. A. souscrits en 1973

\begin{tabular}{l|r}
\hline \multicolumn{1}{c|}{ Pays } & $\begin{array}{r}\text { Pourcentage } \\
\text { des abonnés }\end{array}$ \\
\hline & \\
France & 29,6 \\
C. E. E. & 24,3 \\
Europe de l'Ouest (- C. E. E.) & 13,9 \\
Europe de l'Est & 9,0 \\
Amérique du Nord + Cuba & 12,7 \\
Asie et Océanie & 4,9 \\
Afrique & 5,6 \\
& \\
\hline
\end{tabular}

\section{DISCUSSION}

Le tableau I montre que la longueur moyenne d'un article est de II pages et qu'il en paraît $4^{\circ}$ par an, soit Io par numéro. Il s'agit principalement d'articles originaux (82 p. Ioo) et, les exposés de mise au point ( 12 p. Ioo) ne viennent qu'assez loin derrière.

L'espèce dont l'étude est le plus souvent abordée est la Poule $(26,4$ p. Ioo) suivi par le Bouf (23,9 p. Iоo) puis viennent la méthodologie génétique ( 77,3 p. Iоo), le Porc (Io,2 p. Iоo), la Chèvre (9,6 p. Ioo) et le Mouton (5,6 p. roo), pour toutes les autres espèces regroupées, on ne trouve que 7 p. 100 des articles (tabl. 2).

Le tableau 3 montre que ces espèces " utiles " sont étudiées sous leur aspect pratique puisque 36 p. Ioo environ des articles sont consacrés à la sélection de type quantitatif, et que $46 \mathrm{p}$. Ioo des textes ont été consacrés aux gènes à effets visibles, en vue, pour la plupart, d'application à court terme (le gène de nanisme du Poulet, le culard des bovins). Quant à la caryologie (Io,4 p. IOo des articles), il s'agit là encore d'articles assez orientés puisque le contrôle des chromosomes des reproducteurs tend à se généraliser dans les échanges internationaux de reproducteurs ou de semence.

Le domaine des polymorphismes biochimiques est le parent pauvre (8 p. Ioo des articles), ce qui ne traduit pas évidemment son immense importance théorique mais, outre le fait qu'actuellement on distingue encore mal ses applications en élevage, il existe déjà en Europe plusieurs revues spécialisées.

Un nombre déjà élevé d'auteurs ( $15^{2}$ si l'on compte seulement les articles référencés) ont déjà collaboré à la revue, le tableau 4 montre qu'ils se renouvellent assez rapidement.

Le tableau 5 qui indique que le recrutement des auteurs se fait en France pour plus de 50 p. 100 ou dans la C. E. E. ( 20 p. IOo) mais ces proportions ne traduisent pas exactement le fait qu'un certain nombre d'auteurs recrutés à l'occasion de colloques ou de séminaires, dont la 
revue a assuré la publication, ne sont que des auteurs occasionnels. Comme l'indique le tableau 6 qui fait ressortir que 60 p. Ioo des articles sont signés par au moins un scientifique du Département de Génétique de l'I. N. R. A., et 70 p. Ioo par au moińs un chercheur de l'I. N. R. A. Ainsi s'explique les observations consignées dans le tableau 7 qui font apparaître que près de 82 p. roo des articles sont écrits en français.

Ainsi profilé les A. G. S. A. se classent d'assez loin comme la revue publiant actuellement le plus grand nombre d'articles consacrés à la sélection des espèces zootechniques. Animal Production, organe de la British Society of animal Production sur 82 articles publiés en 1973 n'en consacrait que 23 aux disciplines génétiques. Pour la même année, ce chiffre tombait à I 3 pour Journal of Heredity, 7 pour Journal of Animal Science et 6 pour Zeitschrift für Tierzilchtung und Zuchtungsbiologie.

Les A. G. S. A. sont, à notre connaissance, la seule revue spécialisée de ce type.

Ainsi donc les A. G. S. A. sont animées pour les 3/4 par les chercheurs du Département de Génétique animale de l'I. N. R. A. (au nombre d'une trentaine). Si cette participation correspond au but principal de la revue lors de sa création, on s'est efforcé, dès le début, à ne pas la rendre unique. L'ouverture internationale a été recherchée par des contacts individuels entre chercheurs, par l'organisation de colloques publiés par la revue (par ex. l'intersexualité de la chèvre sans cornes dans le volume I) ou la prise en publication de colloques internationaux comme les Sessions annuelles de la Commission de Génétique de la F. E. Z. (vol. 3, 4, 5) ou la réunion de la Branche européenne de la W. P. S. A. (vol. 4).

Ainsi s'explique l'important taux de renouvellement des auteurs vu plus haut. Cette politique a permis, incontestablement, de retenir des collaborateurs étrangers réguliers. On doit également noter que la langue joue certainement un rôle pour attirer des auteurs francophones (Belges par ex.) ou de langue romane (Italiens).

Une comparaison entre les tableaux 6 et 8 montre que la diffusion dans un pays est à peu près proportionnelle à la contribution des auteurs de ce pays. C'est une justification de la politique d'ouverture évoquée plus haut.

\section{THE " ANNALES DE GÉNÉTIQUE ET DE SÉLECTION ANIMALE » :}

\section{A SUMMARY OF THE FIRST FIVE YEARS}

Founded in 1969, the Annales de Génétique et de Sélection animale are published in an annual volume which has 4 issues. In each volume there are about 40 scientific articles of I I pages. They are mostly original works (82 p. roo).

The Hen is the most studied species (27 p. Ioo of the articles), and next come Cattle (24 p. Ioo of the articles), Pig (10 p. I0o), Goat (9 p. Ioo) and Sheep (6 p. I oo). I 7 p. Ioo of the articles concern genetic methodology and only 7 p. roo of the articles cover other animal species.

Most of the articles treat selection, using either quantitative genetics ( $36 \mathrm{p}$. Ioo) or mendelian genetics. The abundance of articles consecrated to the latter ( 46 p. roo) reflects the present interest of selectioners in visible effect genes in the Hen (dwarfism gene) and in Cattle (double-muscle gene). Caryology is the subject of Io p. Ioo of the articles and Polymorphism 8 p. Ioo. As compared to other current reviews, the Annales de Génétique et de Sélection animale publish the largest number of articles on genetic improvement of domestic species.

The Annales de Génétique et de Sélection animale are published by the Institut national de la Recherche agronomique (I. N. R. A.), and the editorial board is composed of scientists from the Département de Génétique animale which furnished $2 \mathrm{I}$ p. Ioo of the 152 authors already counted and about 60 p. Ioo of the articles. There is a total of 52 p. roo French authors. 82 p. Ioo of the articles are written in French, 16 p. roo in English and 2 p. 100 in German.

Paying subscribers are mostly from France (30 p. I0o) or from the rest of Common Market (24 p. IOo) ; the English-speaking countries, Asia and Africa are not as well covered. 


\section{DIE “ ANNALES DE GÉNÉTIQUE ETT DE SÉLECTION ANIMALE 》 : BILANZ DER ERSTEN FÜNF JAHRE}

Die Annales de Génétique et de Sélection animale (A. G. S. A.), gegründet im Jahre 1969, erscheinen viermal pro Jahr, für ein Band. Jedes Band enthält im Durchschnitt 40 wissenschaftliche Artikel von I I Seiten Länge. Es handelt sich vor allem um Originaltexte (82 p. IOo).

Das Huhn, dem 27 p. I oo der Artikel gewidmet sind, steht an erster Stelle der behandelten Tierarten. Darauf folgen das Rind (24 p. roo der Artikel), das Schwein (1o p. roo), die Ziege (9 p. Ioo und das Schaf (6 p. I0o). I7 p. Ioo der Artikel sind der genetischen Methodik gewidmet, und nur 7 p. Ioo beschäftigen sich mit andern Tierarten.

Die meisten Artikel behandeln die Selektion. Sie stützen sich, entweder auf die quantitative Genetik (zu 36 p. I oo) oder aber auf die mendelsche. Die Fülle der Artikel, die dieser letzteren Disziplin gewidmet sind (46 p. I0o), spiegelt das Interesse wieder, das die Selektioneure den Genen mit sichtbaren Effekten beim Huhn (Zwergwuchsgen) und beim Rind (Doppellendergen) entgegenbringen. Die Caryologie vereinigt Io p. Ioo der Artikel und die Polymorphismen 8 p. Ioo. Unter den gegenwärtigen Fachzeitschriften ist die A. G. S. A. diejenige, welche jährlich am meisten Artikel über die genetische Verbesserung der Haustierarten publiziert.

Die A. G. S. A. werden vom Institut national de la Recherche agronomique (I. N. R. A.) herausgegeben, und ihr Redaktionsausschuss setzt sich aus Forschern des Départements de Génétique animale zusammen, das bis jetzt ebenfalls 2 I p. Ioo der I 52 Autoren gestellt und etwa 60 p. Ioo der Artikel geliefert hat. Man zählt im ganzen $5^{2}$ p. roo französische Autoren. 82 p. Ioo des Artikel sind auf französisch geschrieben, I6 p. Ioo auf englisch und 2 p. Ioo auf deutsch.

Die zu bezahlenden Abonnemente wurden zur Hauptsache in Frankreich bestellt (30 p. Ioo) und auf dem Gebiet des Gemeinsamen Marktes (24 p. I00). Die anglophone Welt, Asien und Afrika sind bis jetzt noch schlecht vertreten. 